\title{
Searches for new phenomena in final states involving leptons and jets using the ATLAS detector
}

\author{
Tadej Novak, on behalf of the ATLAS Collaboration \\ Experimental Particle Physics Department, Jožef Stefan Institute, \\ Jamova cesta 39, 1000 Ljubljana, Slovenia \\ tadej.novak@cern.ch \\ Received XX XX 2020 \\ Revised XX XX 2020
}

\begin{abstract}
Final states containing both leptons and jets can be used to probe for physics beyond the Standard Model. Searches for new physics models with these signatures, such as heavy neutrinos or leptoquarks, for example, are performed using the ATLAS experiment at the LHC. The results of the most recent searches on $13 \mathrm{TeV} p p$ data will be presented.

Keywords: ATLAS; beyond the Standard Model; heavy neutrinos; leptoquarks; seesaw.
\end{abstract}

\section{Introduction}

Searches for physics beyond the Standard Model (SM) can be probed in various different final state topologies at collider experiments. Final states involving both leptons and jets are widely used for searching for specific models yielding increased sensitivity and allowing a larger phase space to be probed.

Several searches using either $36.1 \mathrm{fb}^{-1}$ or $80 \mathrm{fb}^{-1}$ of proton-proton collisions collected by the ATLAS detector ${ }^{1}$ at the Large Hadron Collider (LHC) will be presented. ATLAS is a multi-purpose particle detector with a forward-backward symmetric cylindrical geometry and a near $4 \pi$ coverage in solid angle around the collision point. It consists of an inner tracking detector surrounded by a thin superconducting solenoid, electromagnetic and hadronic calorimeters, and a muon spectrometer incorporating three large superconducting toroidal magnets.

\section{Excited Leptons}

Excited leptons appear in a number of composite models ${ }^{2}$ seeking to explain the existence of the three generations of quarks and leptons in the Standard Model. The composite models introduce new constituent particles called preons that bind at a high scale $\Lambda$ to form SM fermions and their excited states. Excited leptons form vector-like states, $\mathrm{SU}(2)$ doublets and singlets, and acquire masses of the order of $\Lambda$.

The search for excited leptons ${ }^{3}$ uses $36.1 \mathrm{fb}^{-1}$ of ATLAS data collected in 2015 and 2016. The focus of the analysis are excited electrons singly produced in $p p$ 
collisions via a contact interaction $q \bar{q} \rightarrow e e^{*}$ and decaying either to an electron and a pair of quarks $(e q \bar{q})$ through a contact interaction or to a neutrino and a $W$ boson $(\nu W)$ via a gauge interaction, as illustrated in Figure 1. The first channel results in two energetic electrons and at least two hadronic jets $j$, denoted by eejj. In the second channel the $W$ boson decays hadronically, yielding an $e \nu q \bar{q}$ final state. This yields final states with exactly one energetic electron, a large-radius (large- $R$ ) jet $J$ produced by two collimated quarks, and missing transverse momentum, denoted by $e \nu J$.

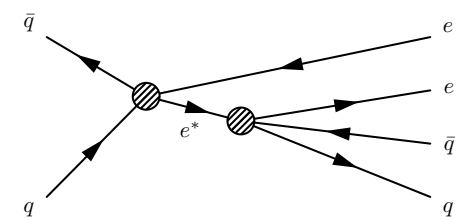

(a)

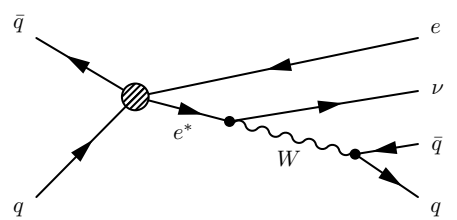

(b)

Fig. 1. Feynman diagrams for (a) $e e^{*} \rightarrow e e q \bar{q}$ and (b) $e e^{*} \rightarrow e \nu W \underline{3}^{3}$

The dominant backgrounds in the eejj and $e \nu J$ channels are from $Z / \gamma^{*}+$ jets and $W+$ jets production, respectively, and are estimated using Monte Carlo simulation. One of the background sources common to both channels is the misidentification of hadronic jets, photon conversions in the material or electrons from hadron decays as prompt electrons, referred to as the fake-electron background. This background is estimated in a data-driven way.

The signal regions for the eejj channel are constructed using the invariant mass of the electron pair $\left(m_{e e}\right)$, the scalar sum of the transverse momenta of the two electrons and the two jets with the highest $p_{\mathrm{T}}\left(H_{\mathrm{T}}\right)$, and the invariant mass of the two electrons and the two jets with the highest $p_{\mathrm{T}}\left(m_{e e j j}\right)$. For the $e \nu J$ channel different discrimination variables have been used, the transverse mass of the system of the missing transverse momentum and the $W$ boson in signal events $\left(m_{\mathrm{T}}^{\nu W}\right)$, and the absolute value of the azimuthal angle between the neutrino and the electron in signal events $\left(\left|\Delta \phi\left(e, E_{\mathrm{T}}^{\mathrm{miss}}\right)\right|\right)$.

The statistical analysis of the search is based on a maximum-likelihood fit. The signal hypothesis test is performed using a likelihood-ratio test statistic in the asymptotic approach.4 No significant excess above the expected SM background is observed, and limits on the excited lepton model parameters are set at $95 \%$ confidence level (CL), using the $\mathrm{CL}_{\mathrm{s}}$ method. For excited electrons with $m_{e^{*}}<1.5 \mathrm{TeV}$, the lower limit on $\Lambda$ is $11 \mathrm{TeV}$, and it decreases to $7 \mathrm{TeV}$ at $m_{e^{*}}=4 \mathrm{TeV}$. In the special case of the excited lepton model where $m_{e^{*}}=\Lambda$, the values of $m_{e^{*}}<4.8 \mathrm{TeV}$ are excluded. Combined lower limits on $\Lambda$ as a function of $m_{e^{*}}$ are displayed in Figure 2 


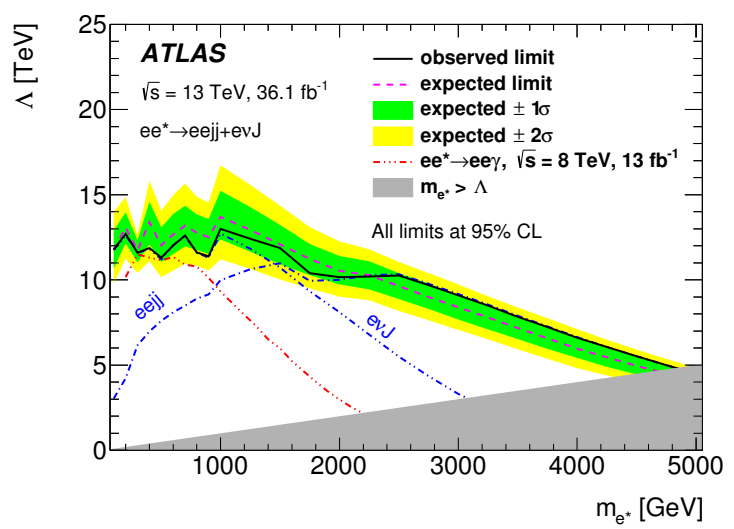

Fig. 2. Combined lower limits on $\Lambda$ as a function of $m_{e^{*}}$. The $\pm 1(2) \sigma$ uncertainty bands around the expected limit represent all sources of systematic and statistical uncertainties. The limits for $m_{e^{*}}>4 \mathrm{TeV}$ are the result of extrapolation $\underline{3}$

\section{Heavy Neutrinos}

Left-right symmetric models (LRSMs) attempt to explain the broken parity symmetry of the weak interaction in the Standard Model and can introduce, depending on the form of the LRSM, right-handed counterparts to the $W$ and $Z$ bosons $\left(W_{\mathrm{R}}\right.$ and $Z_{\mathrm{R}}$ ), and right-handed heavy neutrinos $\left(N_{\mathrm{R}}\right)$. A search for the $W_{\mathrm{R}}$ boson and $N_{\mathrm{R}}$ neutrino production in a final state containing two charged leptons and two jets $(l l j j)$ with $l=e, \mu$ was performed ${ }^{5}$ The specific process of interest is the KeungSenjanović (KS) process, $\frac{6}{6}$ shown in Figure 3. When the $W_{\mathrm{R}}$ boson is heavier than the $N_{\mathrm{R}}$ neutrino $\left(m_{W_{\mathrm{R}}}>m_{N_{\mathrm{R}}}\right)$, the on-shell $W_{\mathrm{R}}$ mass can be reconstructed from the invariant mass of the $\ell \ell j j$ system, whereas, when $\left(m_{W_{\mathrm{R}}}<m_{N_{\mathrm{R}}}\right)$, the on-shell $W_{\mathrm{R}}$ mass can be reconstructed from the invariant mass of the $j j$ system. No mixing between lepton flavours is assumed. In addition to the resolved regime a separate search was performed ${ }^{7}$ focusing on the regime where the $W_{\mathrm{R}}$ is very heavy compared with the $N_{\mathrm{R}}\left(m_{N_{\mathrm{R}}} / m_{W_{\mathrm{R}}} \leq 0.1\right)$. The heavy neutrinos are produced with large transverse momentum (i.e. are highly boosted) and their decay products are very collimated. Therefore a large-radius jet (large- $R$ jet) can be used to reconstruct the $N_{\mathrm{R}}$ so the final state consists only of one lepton and one large-radius jet $(l J)$.

The resolved channel search used $36.1 \mathrm{fb}^{-1}$ of ATLAS data recorded in 2015 and 2016. Heavy neutrinos can either be Dirac (only opposite-charge leptons) or Majorana particles (same-charge final state leptons allowed) which motivated the split of the channel in two sub-channels based on the lepton charge. For the oppositecharge channel, the dominant SM backgrounds are $Z+$ jets and $t \bar{t}$ processes, which are modelled using Monte Carlo simulation. A data-driven $m_{j j}$ reweighting has been applied to improve the modelling of the $Z+$ jets background. Depending on the mass regime described above the reconstructed $W_{\mathrm{R}}$ was used in the fit. In the same-charge channel, the main backgrounds arise from misidentified leptons, 


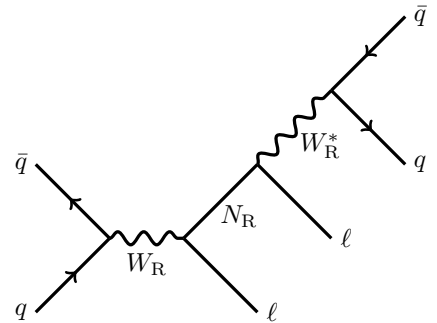

(a)

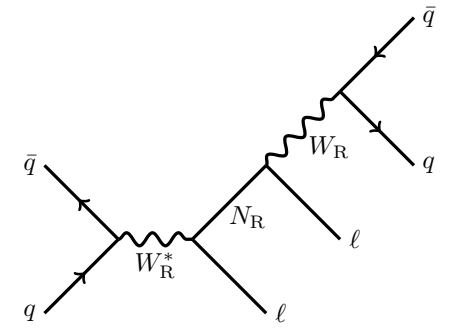

(b)

Fig. 3. Feynman diagrams of the Keung-Senjanović process for (a) $m_{W_{\mathrm{R}}}>m_{N_{\mathrm{R}}}$ case and (b) $m_{W_{\mathrm{R}}}<m_{N_{\mathrm{R}}}$ case $[5$

electron charge misidentification, which are estimated in a data-driven way. The scalar sum of $p_{\mathrm{T}}$ of the two leptons and two leading jets $\left(H_{\mathrm{T}}\right)$ is used in the fit.

The boosted channel search also used 2017 data amounting to an integrated luminosity of $80 \mathrm{fb}^{-1}$. The signal events are selected if they contain exactly two same-flavour leptons (with no charge requirement) and at least one large- $R$ jet with large transverse momentum $p_{\mathrm{T}}>200 \mathrm{GeV}$. The leading lepton should be backto-back in azimuth with the jet, while the subleading lepton should be contained inside the jet. The latter causes $30 \%$ uncertainty on electron identification which was estimated in a different-flavor validation region.

Binned maximum-likelihood fit was performed to obtain limits on masses of $W_{\mathrm{R}}$ and $N_{\mathrm{R}}$. At $95 \%$ confidence level $W_{\mathrm{R}}$ masses are excluded up to $m_{W_{\mathrm{R}}}=4.7 \mathrm{TeV}$ and $N_{\mathrm{R}}$ masses up to $m_{N_{\mathrm{R}}}=3.2 \mathrm{TeV}$ in the resolved channel. The boosted channel additionally improves the limits on $m_{W_{\mathrm{R}}}$ up to $5 \mathrm{TeV}$ for low $m_{N_{\mathrm{R}}}$ as shown in Figure 4

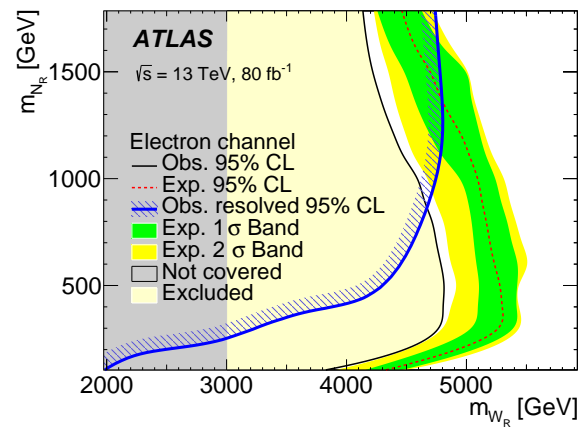

(a)

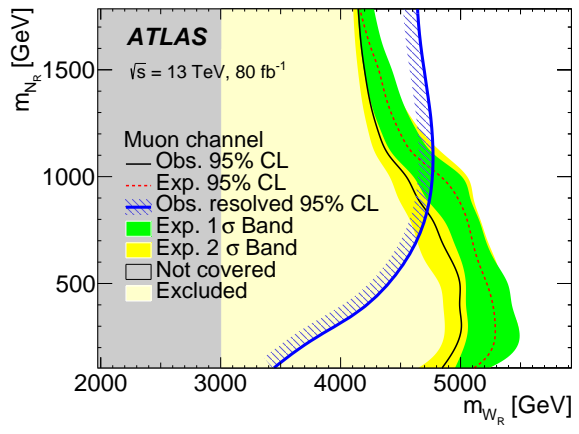

(b)

Fig. 4. Observed (black solid line) and expected (red dashed line) 95\% CL exclusion contours in the $\left(m_{W_{\mathrm{R}}}, m_{N_{\mathrm{R}}}\right)$ plane, along with the $\pm 1 \sigma$ and $\pm 2 \sigma$ uncertainty bands (green and yellow) around the expected exclusion contour in the (a) electron and (b) muon channels. The exclusion limits in the resolved topology are shown by the blue line ${ }^{7}$ 


\section{Scalar Leptoquarks}

Leptoquarks (LQs) are features of a number of extensions of the Standard Model and may provide an explanation for the similarities between the quark and lepton sectors in the SM. They are colour-triplet bosons with fractional electric charge and possess non-zero baryon and lepton number ${ }^{8}$ Scalar and vector LQs have been proposed and are expected to decay directly into lepton-quark pairs. The lepton can be either electrically charged or neutral. The benchmark signal model used for this search is the minimal Buchmüller-Rückl-Wyler (BRW) model ${ }^{[9}$ LQs belong to three families, corresponding to the three SM generations, such that only leptons and quarks within a given generation can interact. The couplings are determined by two parameters: a model parameter $\beta$ and the coupling parameter $\lambda$. The coupling to the charged lepton is given by $\sqrt{\beta} \lambda$, and the coupling to the neutrino by $\sqrt{1-\beta} \lambda$. The branching ratio $B$ into charged leptons is for first and second generations directly proportional to $\beta$, but it also has leptoquark mass dependance for third generation due to sizeable top-quark mass.

The search focused on pair production of scalar leptoquarks using $36.1 \mathrm{fb}^{-1}$ of ATLAS data. It was split into several channels primarily split between first and second generations, $\frac{10}{10}$ and third generation leptoquarks. 11 The light generations channel is focusing on two different final state topologies, one consisting of two leptons and at least two jets $\ell \ell j j$, and the other of exactly one lepton, missing transverse momentum and at least two jets $\ell \nu j j$. The electron and muon channels are treated separately as they correspond to LQs of different generations.

The third generation searches are all reinterpretations of existing ATLAS searches. One is based on a search for pair-produced Higgs bosons decaying into two $b$-jets and two $\tau$-leptons, and four are reinterpretation searches for supersymmetric particles. They cover topologies with two top-quark decays and $E_{\mathrm{T}}^{\text {miss }}$, two $\tau$-leptons one $b$-jet and $E_{\mathrm{T}}^{\mathrm{miss}}$, and two $b$-jets and $E_{\mathrm{T}}^{\mathrm{miss}}$. Supersymmetric particles can have similar or even identical experimental signatures and very similar kine-

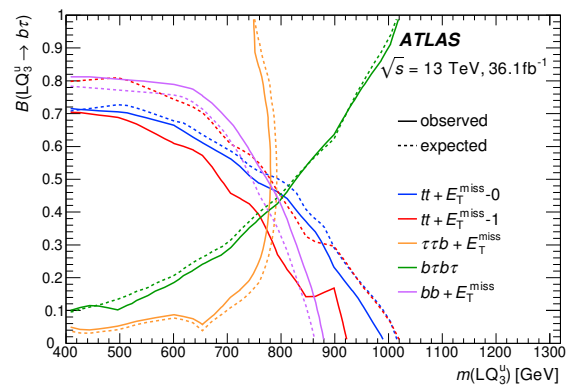

(a)

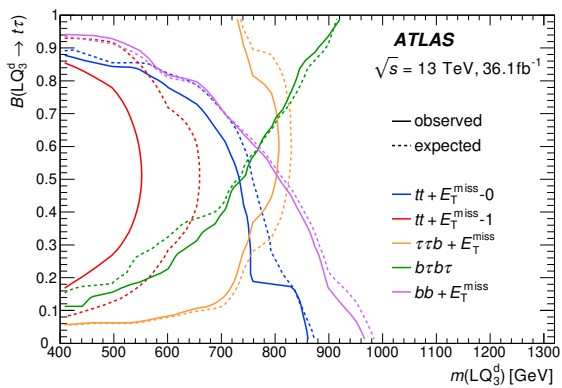

(b)

Fig. 5. Limits on the branching ratio into charged leptons for scalar third generation (a) up-type leptoquark pair production and (b) down-type leptoquark pair production as a function of the leptoquark mass 11 
matics to pair-produced LQs.

The observed and expected lower limits on the leptoquark mass at $95 \%$ confidence level extend up to $1.25 \mathrm{TeV}$ for first and second generation leptoquarks. A separate limit was set for each of the channels of the third generation search as shown in Figure 5. LQ masses below $0.8 \mathrm{TeV}$ are excluded independently of the branching ratio $B$. For the special cases of $B=0$ and $B=1$ third generation scalar leptoquarks are excluded up to $1 \mathrm{TeV}$.

\section{Dijet Resonances in Events With an Isolated Lepton}

Searches for resonances in dijet invariant mass distributions provide a means to investigate a wide range of theories beyond the Standard Model (BSM). Such searches are sensitive to heavy particles that decay into two partons which, following hadronization, form two jets. Selecting events with a final-state lepton in addition to dijets increases sensitivity to a different range of exotic models. In addition, exploitation of the lepton in event selection gives an opportunity to probe smaller dijet invariant masses than in the previous dijet searches based on jet triggers.

The study presented focuses on a generic search ${ }^{12}$ for BSM resonances in the dijet invariant mass distribution constructed from events with at least one isolated lepton (muon or electron) and uses an integrated luminosity of $80 \mathrm{fb}^{-1}$ of ATLAS data. The presence of a new resonance with mass $m_{X}$ may lead to an excess of events at $m_{j j}=m_{X}$ in an otherwise smooth and monotonically decreasing dijet invariant mass distribution. The background shape was fitted with a function

$$
f(x)=p_{1}(1-x)^{p_{2}} x^{p_{3}+p_{4} \ln x+p_{5} \ln ^{2} x},
$$

where $x \equiv m_{j j} / \sqrt{s}$ and the $p_{i}$ are five free parameters to be estimated.

In order to determine the most significant deviation from the background only hypothesis, the BumpHunter $\frac{13}{13}(\mathrm{BH})$ test is used. In the $\mathrm{BH}$ procedure, for each of the $\mathrm{N}$ possible intervals in $m_{j j}$, a local $p$-value is calculated from the backgroundonly hypothesis test statistic. The result of the background fit and the $\mathrm{BH}$ procedure is shown on Figure 6 with the highest deviation at $m_{j j}=3.56 \mathrm{TeV}$ corresponding to a significance of 2.5 standard deviations. In the absence of any significant excess, upper limits have been calculated for different resonance widths, from a narrow width to $15 \%$ of the mean mass. The resulting limits range from $50 \mathrm{fb}$ to $0.1 \mathrm{fb}$ in the mass range from 0.25 to $6 \mathrm{TeV}$ and are presented in Figure 7 .

\section{Type-III seesaw heavy leptons}

The experimental observation of neutrino oscillation shows that neutrinos have non-zero masses, which are much smaller than those of the charged leptons (see Ref. 14 and references therein). The ATLAS search using $80 \mathrm{fb}^{-1}$ of data presented at this conference ${ }^{155}$ uses a minimal type-III seesaw mode ${ }^{[16}$ focusing on the lightest 


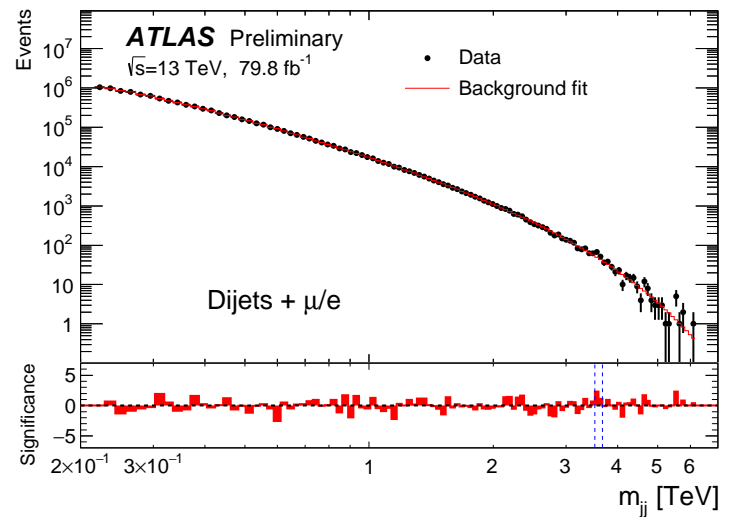

Fig. 6. The dijet mass distribution for events with leptons of $p_{\mathrm{T}}>60 \mathrm{GeV}$. The data are shown together with the background fit (shown with the red line). The fit corresponds to $\chi^{2} / \mathrm{ndf}=1.02$ in the considered mass range. The lower panel shows the bin-by-bin significances of the data-fit differences considering only statistical uncertainties 12

fermionic triplet of unknown (heavy) masses with one neutral and two oppositelycharged leptons denoted by $\left(L^{+}, L^{-}, N^{0}\right)$. Here $L^{+}$is the antiparticle of $L^{-}$and $N^{0}$ is a Majorana particle. These heavy leptons decay into a SM lepton and a $W, Z$ or $H$ boson. The heavy leptons are assumed to be degenerate in mass $\frac{17}{17}$ and the decay branching ratios are considered equal for all three lepton flavors. Pair-production through $p p \rightarrow W^{*} \rightarrow N^{0} L^{ \pm}$with one of the two $W$ bosons decaying leptonically and the other decaying hadronically was considered, as illustrated in Figure 8 . The

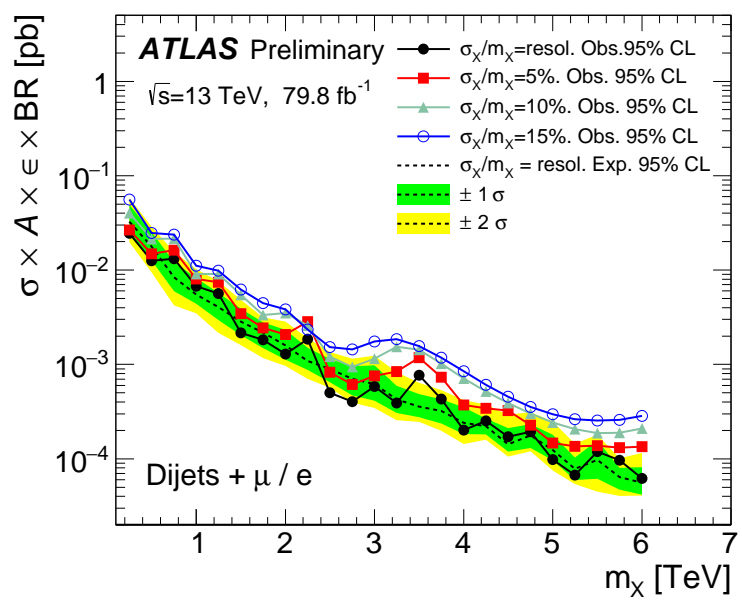

Fig. 7. Comparisons of the $95 \%$ credibility-level upper limits for a BSM signal approximated by Gaussian contributions with different widths. The limits are weaker for broader resonances for which Gaussian contributions span more $m_{j j}$ bins 12 


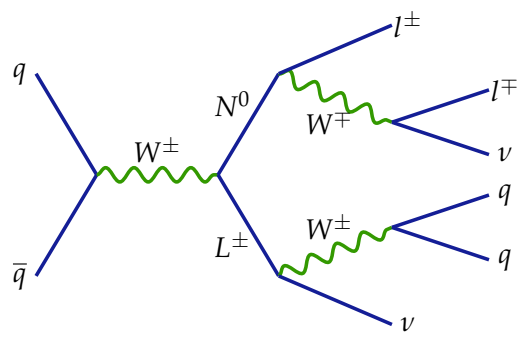

(a)

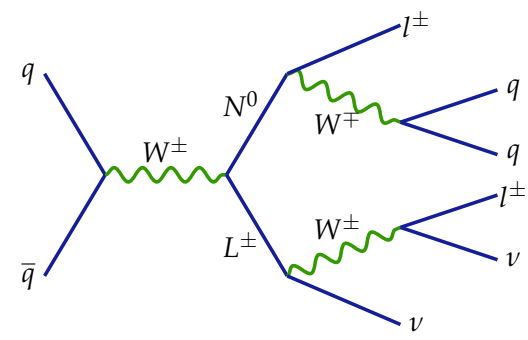

(b)

Fig. 8. Feynman diagrams of the considered type-III seesaw production process in the (a) OS and (b) SS final state cases 15

exclusive topology of the final state consists of two jets from the $W$ boson, large missing transverse momentum $\left(E_{\mathrm{T}}^{\mathrm{miss}}\right)$ and a lepton pair with either the same-sign charge (SS) or with the opposite-sign charge (OS) and with either same-flavor (ee or $\mu \mu)$ or different-flavor $(e \mu)$ combinations.

The six OS and SS analysis channels are defined according to the flavor combination. Different selection criteria are applied to ensure the highest possible signal and background separation. The invariant dijet mass $\left(m_{j j}\right)$ of the two leading jets is expected to be close to the $W$ mass. As the signal process contains neutrinos in the final state, one of the most important selection criteria is based on the $E_{\mathrm{T}}^{\mathrm{miss}}$ significance $\operatorname{Sig}\left(E_{\mathrm{T}}^{\mathrm{miss}}\right) !{ }^{18}$ In the OS channels the azimuthal angle $\Delta \phi\left(E_{\mathrm{T}}^{\mathrm{miss}}, l\right)_{\text {min }}$ between the directions of transverse momenta of $E_{\mathrm{T}}^{\mathrm{miss}}$ and closest lepton has a very good separation power, exploiting the different nature of $E_{\mathrm{T}}^{\mathrm{miss}}$ between signal and background.

A binned maximum-likelihood fit of the $H_{\mathrm{T}}+E_{\mathrm{T}}^{\text {miss }}$ variable distribution, the sum of the $E_{\mathrm{T}}^{\mathrm{miss}}$ and the scalar sum of the transverse momenta $H_{\mathrm{T}}$, was used to

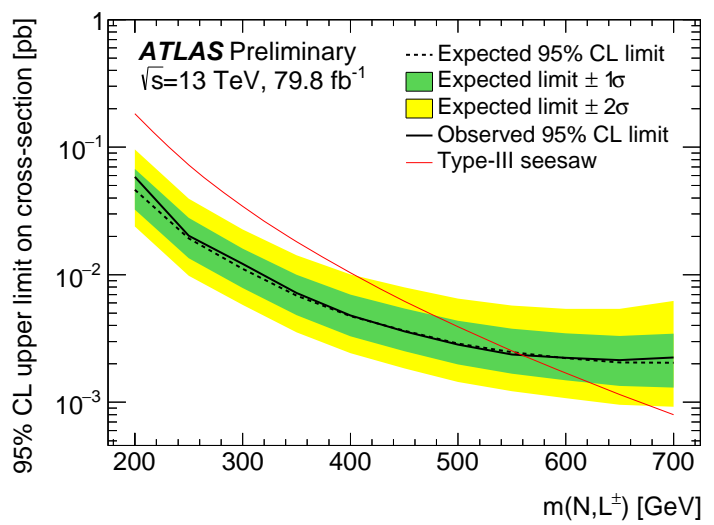

Fig. 9. Expected and observed 95\% CLs exclusion limits on the type-III seesaw cross section with the corresponding one and two standard deviation bands 15 
obtain the numbers of signal and background events. The compatibility between the data and the expected background is assessed and good agreement is observed. $95 \%$ confidence level upper limits were derived as a function of the heavy lepton mass and are shown on Figure 9. The type-III seesaw heavy leptons observed limit has been placed at $560 \mathrm{GeV}$, excluding the mass values below this point.

\section{Conclusions}

The ATLAS experiment at the LHC has performed many searches with leptons and jets in the final state using $36.1 \mathrm{fb}^{-1}$ and $80 \mathrm{fb}^{-1}$ of collected data. No significant excess from the Standard Model has been observed and limits on production cross sections of various theories beyond the Standard Model have been set.

\section{References}

1. ATLAS Collaboration, JINST 3, S08003 (2008), doi:10.1088/1748-0221/3/08/S08003.

2. U. Baur, M. Spira and P. Zerwas, Phys.Rev.D 42, 815 (1990), doi:10.1103/PhysRevD. 42.815 .

3. ATLAS Collaboration, Eur.Phys.J.C 79, 803 (2019), arXiv:1906.03204 [hep-ex], doi:10.1140/epjc/s10052-019-7295-1.

4. G. Cowan, K. Cranmer, E. Gross and O. Vitells, Eur.Phys.J.C 71, 1554 (2011), arXiv:1007.1727 [physics.data-an], doi:10.1140/epjc/s10052-011-1554-0, [Erratum: Eur.Phys.J.C 73, 2501 (2013)].

5. ATLAS Collaboration, JHEP 01, 016 (2019), arXiv:1809.11105 [hep-ex], doi:10. 1007/JHEP01(2019)016.

6. W.-Y. Keung and G. Senjanovic, Phys.Rev.Lett. 50, 1427 (1983), doi:10.1103/ PhysRevLett.50.1427.

7. ATLAS Collaboration, Phys.Lett.B 798, 134942 (2019), arXiv: 1904.12679 [hep-ex], doi:10.1016/j.physletb.2019.134942.

8. B. Schrempp and F. Schrempp, Phys.Lett.B 153, 101 (1985), doi:10.1016/ 0370-2693(85)91450-9.

9. W. Buchmuller, R. Ruckl and D. Wyler, Phys.Lett.B 191, 442 (1987), doi:10.1016/ 0370-2693(87)90637-X, [Erratum: Phys.Lett.B 448, 320-320 (1999)].

10. ATLAS Collaboration, Eur.Phys.J.C 79, 733 (2019), arXiv:1902.00377 [hep-ex], doi:10.1140/epjc/s10052-019-7181-x.

11. ATLAS Collaboration, JHEP 06, 144 (2019), arXiv:1902.08103 [hep-ex], doi:10. 1007/JHEP06(2019)144.

12. ATLAS Collaboration, Search for dijet resonances in events with an isolated lepton using $\sqrt{s}=13 \mathrm{TeV}$ proton-proton collision data collected by the ATLAS detector, ATLAS-CONF-2018-015 (Jun 2018), http://cds.cern.ch/record/2621126.

13. G. Choudalakis, On hypothesis testing, trials factor, hypertests and the bumphunter, in Proceedings, PHYSTAT 2011 Workshop on Statistical Issues Related to Discovery Claims in Search Experiments and Unfolding, CERN,Geneva, Switzerland 17-20 January 2011, arXiv:1101.0390 [physics.data-an].

14. Particle Data Group Collaboration (C. Patrignani et al.), Chin. Phys. C40, 100001 (2016), doi:10.1088/1674-1137/40/10/100001.

15. ATLAS Collaboration, Search for type-III seesaw heavy leptons in proton-proton collisions at $\sqrt{s}=13 \mathrm{TeV}$ with the ATLAS detector, ATLAS-CONF-2018-020 (Jun 2018), http://cds.cern.ch/record/2621484. 
16. C. Biggio and F. Bonnet, Eur. Phys. J. C72, 1899 (2012), arXiv:1107.3463 [hep-ph], doi:10.1140/epjc/s10052-012-1899-z.

17. R. Franceschini, T. Hambye and A. Strumia, Phys. Rev. D78, 033002 (2008), arXiv:0805.1613 [hep-ph], doi:10.1103/PhysRevD.78.033002.

18. ATLAS Collaboration, Eur. Phys. J. C 78, 903 (2018), arXiv:1802.08168 [hep-ex], doi:10.1140/epjc/s10052-018-6288-9. 